Provided for non-commercial research and education use. Not for reproduction, distribution or commercial use.

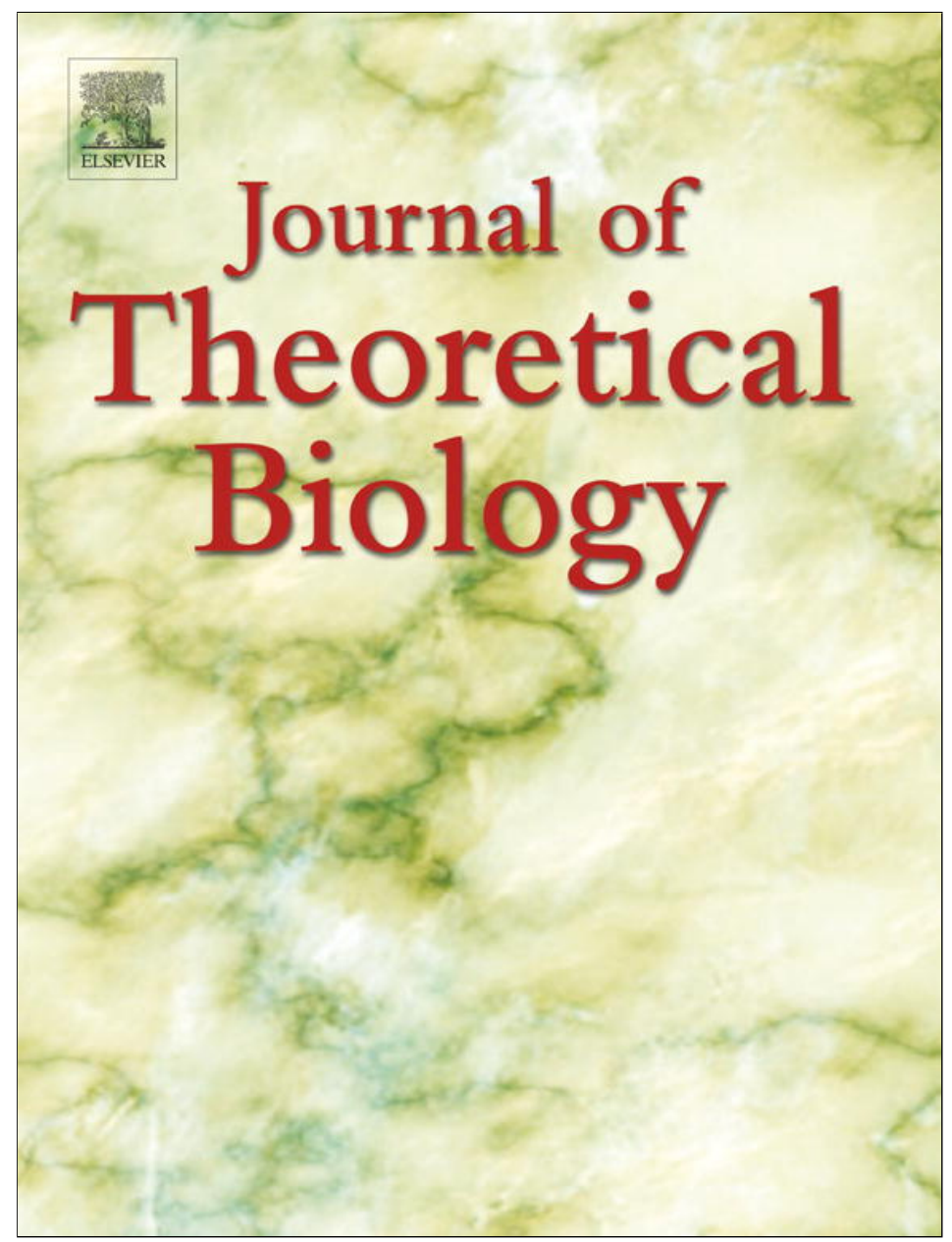

(This is a sample cover image for this issue. The actual cover is not yet available at this time.)

This article appeared in a journal published by Elsevier. The attached copy is furnished to the author for internal non-commercial research and education use, including for instruction at the authors institution and sharing with colleagues.

Other uses, including reproduction and distribution, or selling or licensing copies, or posting to personal, institutional or third party websites are prohibited.

In most cases authors are permitted to post their version of the article (e.g. in Word or Tex form) to their personal website or institutional repository. Authors requiring further information regarding Elsevier's archiving and manuscript policies are encouraged to visit: 


\title{
Modelling the within-host growth of viral infections in insects
}

\author{
S.M. White ${ }^{\mathrm{a}, \mathrm{b}, *}$, J.P. Burden ${ }^{\text {a }}$, P.K. Maini ${ }^{\mathrm{b}, \mathrm{c}}$, R.S. Hails ${ }^{\mathrm{a}}$ \\ ${ }^{a}$ Centre for Ecology \& Hydrology, Maclean Building, Benson Lane, Crowmarsh Gifford, Wallingford, Oxfordshire OX10 8BB, United Kingdom \\ ${ }^{\mathrm{b}}$ Centre for Mathematical Biology, Mathematical Institute, University of Oxford, 24-29 St Giles', Oxford, Oxfordshire OX1 3LB, United Kingdom \\ ${ }^{\mathrm{c}}$ Oxford Centre for Integrative Systems Biology, Department of Biochemistry, South Parks Road, Oxford OX1 3QU, United Kingdom
}

\section{H I G H L I G H T S}

- We study the within-host growth of obligate-killing insect viruses.

- We assay four virus strains and fit statistical and mathematical models to the data.

- Statistical analyses reveal differences in the growth rate of the host and viruses.

- We show that initially the viruses grow at a double exponential rate.

- Our mathematical model predicts a decrease in viral yield as dose increases.

\section{A R T I C L E I N F O}

\section{Article history:}

Received 19 December 2011

Received in revised form

16 May 2012

Accepted 23 July 2012

Available online 31 July 2012

Keywords:

Lepidoptera

Nucleopolyhedrovirus

Consumer-resource dynamics

Biocontrol

Baculovirus

\begin{abstract}
A B S T R A C T
Insects are infected by a variety of pathogens, including bacteria, fungi and viruses, which have been studied largely for their potential as biocontrol agents, but are also important in insect conservation (biodiversity) and as model systems for other diseases. Whilst the dynamics of host-pathogen interactions are well-studied at the population level, less attention has been paid to the critical within-host infection stage. Here, the reproductive rate of the pathogen is largely determined by how it exploits the host; the resources supplied by the host in terms of size and condition; competition with other pathogens; and the speed with which it kills the host (death being an inevitable outcome for obligate-killing pathogens). In this paper we aim to build upon recent developments in the literature by conducting single infection bioassays to obtain data on growth and fitness parameters for phenotypically different and similar strains of nucleopolyhedroviruses in the Lepdipoteran host Spodoptera exigua. Using these data, a simple mechanistic mathematical model (a coupled system of differential equations) is derived, fitted and parameter sensitivity predictions are made which support empirical findings. We unexpectedly found that initial growth of virus within the host occurs at a doubleexponential rate, which contrasts with empirical findings for vertebrate host-pathogen systems. Moreover, these infection rates differ between strains, which has significant implications for the evolution of virulence and strain coexistence in the field, which are still relative unknowns. Furthermore, our model predicts that, counter to intuition, increased viral doses may lead to a decrease in viral yield, which is supported by other studies. We explain the mechanism for this phenomenon and discuss its implications for insect host-pathogen ecology.
\end{abstract}

(c) 2012 Elsevier Ltd. All rights reserved.

\section{Introduction}

Pathogens play an important role for many host organisms, ranging from population regulation (Hudson et al., 1998) to species invasion (Borer et al., 2007). These in turn, have applications for our understanding of issues such as disease control

\footnotetext{
* Corresponding author at: Centre for Ecology \& Hydrology, Maclean Building Benson Lane, Crowmarsh Gifford, Wallingford, Oxfordshire OX10 8BB, United Kingdom. Tel.: + 441491 692699; fax: +44 1491692424 .

E-mail addresses: smwhit@ceh.ac.uk (S.M. White), jbur@ceh.ac.uk (J.P. Burden), maini@maths.ox.ac.uk (P.K. Maini), rha@ceh.ac.uk (R.S. Hails).
}

(Blanford et al., 2005), pest control (Mitchell and Power, 2003) and biodiversity (Daszak et al., 2000). However, much of our empirical understanding of host-pathogen ecology and evolution is derived at the population level (see Dwyer et al., 2000 for example), and the crucial pathogen stages operating within the host are either simply over-looked or assumed to be nondynamic, whereas, in reality, key pathogen fitness and virulence traits are often determined throughout the course of infection (e.g. environmental conditions). For example, in the case of monarch butterflies, Danaus plexippus, rearing infected larvae on different plant species alters parasite infection, replication and virulence (de Roode et al., 2008). This illustrates that population 
dynamics of hosts and pathogens will be subjected to feedback mechanisms from the pathogen dynamics within the host.

This point is now being addressed by a number of authors, especially via theoretical means. For example, Antia and Lipsitch (1997) proposed a mathematical model for an acute microparasite infection in a vertebrate host. This model suggested that the within-host dynamics of the microparasite will be a 'race' between parasite multiplication and the clonally expanding response by the host immune system, resulting either in immune-mediated clearance or in host death. In a mathematically similar, but biologically different system, Ellner et al. (2007) modelled the within-host interaction of a fungal pathogen in a coral. Here the fungal-immune system dynamics are rather complex and spatially explicit, highlighting the importance of 'immune response free space' which allows local rapid growth of the fungal infection. One application of these types of models has been to improve our understanding of pathogen evolution, which has revealed that the dynamics of the immune system may select for parasites with intermediate within-host growth rates, as this is when the number of transmission stages from infected hosts reaches a maximum (Antia et al., 1994).

A large proportion of previous theoretical models based the infection dynamics on Lotka-Volterra interactions (see Alizon and van Baalen, 2008 for an example and references therein) or models with a fixed kill rate by immunity (Perelson, 2002) and are aimed at vertebrate hosts, largely due to the applicability to human health. In contrast, little work has been carried out for invertebrate systems, particularly with empirical data to test the model.

The dynamics of pathogens within-invertebrate hosts differ significantly from those of vertebrates. Firstly, many insect pathogens are obligate killers, in the sense that effective horizontal transmission may only be attained by the death of the host, whereas for most vertebrates the infectious stages are emitted throughout the course of infection. Secondly, since the host itself is simply a resource for the virus to reproduce, the size and growth rate of the host is crucial in determining the speed of pathogen replication and the yield of infectious stages. Lastly, common to both vertebrates and invertebrates, are innate immune mechanisms that can be either constitutively expressed or induced on exposure to infection (Hamilton et al., 2008). However, invertebrates lack acquired immune responses, but their mechanistically simple innate effectors are functionally sophisticated and can be highly efficient (Siva-Jothy et al., 2005). Therefore, the vertebrate models do not lend themselves readily to invertebrate systems.

Ebert and Weisser (1997) proposed a model for the dynamics of the within-host growth of obligate-killing parasites, such as baculoviruses, and many species of bacteria, bacteriophages, nematodes, fungi and microsporidia. Their model assumes that pathogen biomass grows logistically, where the carrying capacity (invertebrate host mass) is time-dependent (also assumed to be logistic in growth) and crucially does not depend on the extent of infection within the host. It is possible, however, that as the pathogen spreads through host tissues it would interfere with metabolic processes and ultimately inhibit the growth of the host. In this case, the host size at the time of death, and therefore the 'pathogen carrying capacity' of the host, should depend on the extent of host tissue infection. Typically, this is not the case in vertebrate infections, and so the interaction between virus replication and host growth rates has not been explicitly considered. In this paper we aim to address this by developing a more biologically detailed model for the within-host growth of obligate killing viruses of invertebrates, which is parameterised and validated against experimental data.

We base our model on baculoviruses, a group of double stranded DNA obligate killing viruses, which have been particularly well studied because of their utility as expression vectors and biocontrol agents (Lacey and Kaya, 2007). Baculoviruses can be subdivided into two distinct genera, Granuloviruses (GVs) and Nucleopolyhedroviruses (NPVs - the focus of this paper), and are indirectly transmitted pathogens, persisting outside their arthropod hosts as occlusion bodies (OBs), a proteinaceous matrix in which the virus particles are embedded. The OBs may contain many virus genomes. Hosts (primarily Lepidoptera) become infected by consuming OBs when eating foliage. The protein dissolves in the alkaline gut of the caterpillar allowing viruses to cross the gut wall and then to start replicating. Overt infections result in the death of the host a few days later. Body tissues are then dissolved with millions of virus particles being produced as a result. These OBs persist in the environment until consumed by a new host or are degraded by environmental factors.

In this paper, we begin by describing bioassays carried out with lepidopteran hosts, in which we determine key life-history traits of the baculovirus and the within-host growth rate of the different strains of virus. We empirically explore the possibility that the virus infection may impede host growth rate, and whether this inhibition increases as the infection progresses using statistical models. We then develop a novel mathematical model for the within-host growth of the different strains of virus. This model incorporates the interactions observed and is parameterised by experimental data. The ecological implications are then discussed.

\section{Infection bioassays and results}

\subsection{Materials E' methods}

\subsubsection{Insect and virus stocks}

Spodoptera exigua larvae were reared in continuous culture on artificial diet (Hunter-Fujita et al., 1998). Four different baculoviruses were used in this study; the Oxford strain of Mamestra brassicae nucleopolyhedrovirus ( $M b$ NPV) (Burden et al., 2006), Panolis flammea nucleopolyhedrovirus (Pafl NPV) variant 4 (Hodgson et al., 2001), Autographa californica nucleopolyhedrovirus (Ac NPV) strain C6 (Ayres et al., 1994) and Spodoptera exigua nucleopolyhedrovirus (Se NPV) (Ijkel et al., 1999). Additional details can be found in the electronic supplementary material (ESM).

\subsubsection{Determination of median lethal dose and mean time to death}

Three blocked bioassays were carried out to determine the median lethal concentration ( $\left.\mathrm{LC}_{50}\right)$ and mean time to death of the four viruses in $S$. exigua. Newly moulted third instar larvae of $S$. exigua were selected on the basis of head capsule diameter and starved overnight at $28^{\circ} \mathrm{C}$. Thirty insects per treatment were then dosed by droplet feeding (Hughes et al., 1986) with $1 \mu \mathrm{l}$ of the virus concentrations specified. The time taken to administer each treatment was recorded and the start time $\left(T_{0}\right)$ taken as the midpoint of this. The exposed larvae were transferred to individual pots of artificial diet and reared at $28{ }^{\circ} \mathrm{C}$ and checked after $24 \mathrm{~h}$, at which point any handling deaths were removed. The larvae were subsequently checked every $12 \mathrm{~h}$ until death or pupation (if the host survived infection) and any levels of mortality and time to death recorded. Details of our statistical methods can be found in the ESM.

\subsubsection{Measurement of the within host-growth of baculoviruses}

Based on the data generated in the previous bioassays a virus concentration of $1 \times 10^{7} \mathrm{OBs} / \mathrm{ml}$ was selected for all four viruses as at this dose all insects should be infected. Newly moulted third instar S. exigua larvae were starved overnight and 200 larvae dosed with $1 \mu$ l of either Ac NPV, Mb NPV, Pafl NPV or Se NPV virus 
at a concentration of $1 \times 10^{7} \mathrm{OBs} / \mathrm{ml}$. The larvae were transferred to individual pots of artificial diet and reared at $28{ }^{\circ} \mathrm{C}$. After $2 \mathrm{~h} 10$ larvae were collected from each treatment. These were then weighed and frozen at $-20^{\circ} \mathrm{C}$ until DNA extraction. The process of weighing and freezing 10 individual larvae was repeated at $12 \mathrm{~h}$ intervals until all remaining larvae had died from virus infection.

Details of the DNA extraction and quantification can be found in the ESM.

\subsection{Bioassay results}

2.2.1. Infectivity and speed of kill of Ac NPV, Mb NPV, Pafl NPV and Se NPV in third instar larvae of S. exigua

The mortality of third instar larvae of $S$. exigua was significantly different between the viruses $\left(\chi^{2}=98.1, \mathrm{df}=4, p \leq 0.001\right)$ although there was no significant difference between the mortality induced by $A c \mathrm{NPV}$ and $M b \operatorname{NPV}\left(\chi^{2}=1.42, \mathrm{df}=1, p=0.233\right)$ (see Fig. S1(a) in the ESM). The mortality was significantly affected by dose of virus $\left(\chi^{2}=185.4, \mathrm{df}=1, p<0.001\right)$ but there was no significant interaction between dose and virus $\left(\chi^{2}=6.59\right.$, $\mathrm{df}=4, p=0.159)$. Overall Se NPV showed the highest mortality and Pafl NPV showed the lowest mortality, and in all cases mortality increased with dose.

Time to death was significantly different between the viruses (see Fig. S1(b) in the ESM), with a significant interaction between virus dose and virus strain $\left(F_{4,799}=16.59, p \leq 0.001\right)$. The time to death of Ac NPV, Mb NPV and Se NPV decreased with increasing virus dose, however the slope of the line for Pafl NPV was not significantly different from zero $\left(F_{2,797}=1.355, p=0.259\right)$ showing that the speed of kill of this virus was unaffected by dose.

\subsubsection{Host growth and the within-host growth of baculoviruses in} third instar larvae of $S$. exigua

Host weight showed distinct differences between infected and uninfected insects (see Fig. 1). Growth rates are curvilinear with time (minimally adequate statistical models), and the degree of this curvilinearity varies with virus strain (virus*time ${ }^{3}$, $F_{4,594}=3.41, p=0.009$ ) indicating that different strains impede host growth to varying degrees. The uninfected larvae grow to their peak in mass before a decrease in weight due to larvae preparing for pupation (Fig. 1(e)). All infected larvae are smaller in mass in comparison with uninfected larvae, particularly at the later stages of infection prior to virus induced death. Both Ac NPV and Mb NPV infected larvae showed a similar decrease in host mass to controls at the latter stages of infection (Fig. 1(a) and (b)), but no such effect was shown for Pafl NPV and Se NPV (Fig. 1(c) and (d)).

Taking the first seven census host growth data points from each treatment, when the $\log _{10}$ weight grows linearly with time (Fig. 1(f) - minimally adequate statistical model), the infected hosts (as one category) show a significantly slower initial growth rate than their uninfected counterparts $\left(F_{1,345}=6.813, p=0.009\right)$. Furthermore, the growth rates of control and infected larvae were individually compared (virus*time, $F_{4,339}=4.617, p=0.001$ ) indicating that NPV viral infections alter the growth of the host differentially during the early stages of infection. Pafl NPV and Se NPV infected larvae showed significantly reduced initial growth rates compared to the uninfected larvae (virus*time, $t_{1,339}=3.645, p=0.0003$ and $t_{1,339}=3.039, p=0.003$, respectively). Interestingly, Ac NPV and $M b$ NPV infected individuals showed no significant difference in initial growth rates when compared to their uninfected conspecifics.

The growth of the viruses within S. exigua, as measured by the proportion of total DNA represented by viral DNA, also varied significantly between the four viruses (see Fig. 2). This relationship is highly non-linear and the degree of non-linearity varies (virus*time ${ }^{5}, F_{3,594}=3.19, p=0.025$ ). All viral treatments showed a $\log$ sigmoidal relationship with time, with all treatments approaching an asymptotic proportion of DNA. Moreover, all treatments also showed a decrease in the proportion of viral DNA shortly after inoculation, with Se NPV and Ac NPV showing the greatest reduction (approximately a 10 -fold reduction). The four viruses also showed differences in the maximum proportion of the host they converted to virus biomass. Ac NPV had the highest ratio of virus to host DNA with a peak of $45 \%$. Se NPV was unable to replicate as much viral DNA, peaking at $12.5 \% \mathrm{Mb}$ NPV and Pafl NPV had the slowest speeds of kill and the lowest proportion of viral DNA ( $8 \%$ and 10\% respectively).

\section{Within-host virus growth mathematical model}

Using the statistical model fitting above we have been able to demonstrate differences in the growth dynamics of the four strains of virus within the host. However, this analysis does not inform us of the importance of various mechanisms and factors of viral infection. To address this we derive the mathematical model below.

\subsection{The model}

Let $H(t)$ and $V(t)$ be the mass of healthy host tissue and mass of virus within the host at time $t$, respectively. We assume that the host is an invertebrate and thus has no acquired immunity (SchmidHempel, 2005). Here, we only consider overt infections where the initial dose of virus is sufficiently large such that the innate immune response is negligible and cannot clear the infection, leading to host death. The host grows with growth rate $r(t)$. Note that since we are only interested in overt infections, it is not necessary to consider host growth in the absence of infection, where the dynamics are considerably different (e.g. overt infections will prevent the onset of pupation). Healthy host mass is infected and converted into virus mass according to the mass action law with a transmission coefficient $\beta(t)$. Here, the assumption is that all infected host tissue is converted into virus (to the best of our knowledge, it is not known if at the cellular level infected host cells produce 'waste', thus warranting a conversion efficiency parameter). These simplifying assumptions lead to the following model:

$\frac{\mathrm{d} H}{\mathrm{~d} t}=r(t) H-\beta(t) H V$

$\frac{\mathrm{d} V}{\mathrm{~d} t}=\beta(t) H V$

where $H(t), V(t)>0$ for all $t \geq 0$.

During the course of infection, the host becomes increasingly moribund and in the latter stages of infection the host almost completely stops eating and therefore stops growing. This is demonstrated in Section 2.2.2 and supported by additional and closely related findings (Burand and Park, 1992). Furthermore, we assume that increased viral loads will have greater effect on the host growth rate (Burand and Park, 1992). We model this by the following integral equation

$r(t)=r_{0} \exp \left(-a \int_{0}^{t} V(s) \mathrm{d} s\right)$

where $r_{0}$ is the maximum host growth rate and $a$ is the host growth reduction rate. Here, the growth rate decreases with the 'experience' of the infection. Note that for mathematical and numerical analyses it is useful to differentiate (1c) with respect to time. 
a

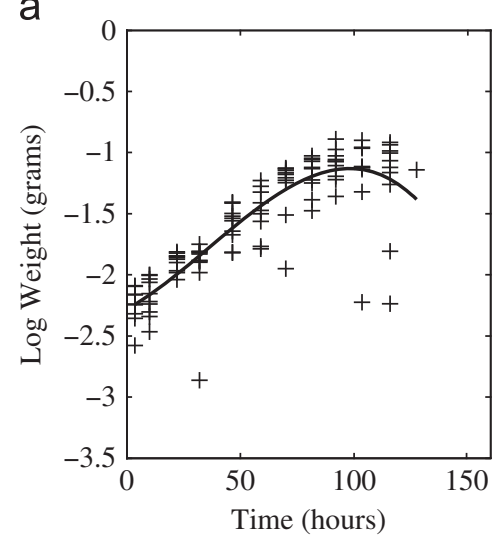

d

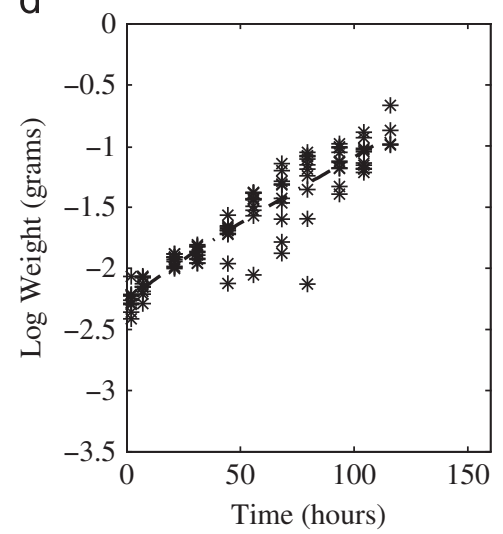

b

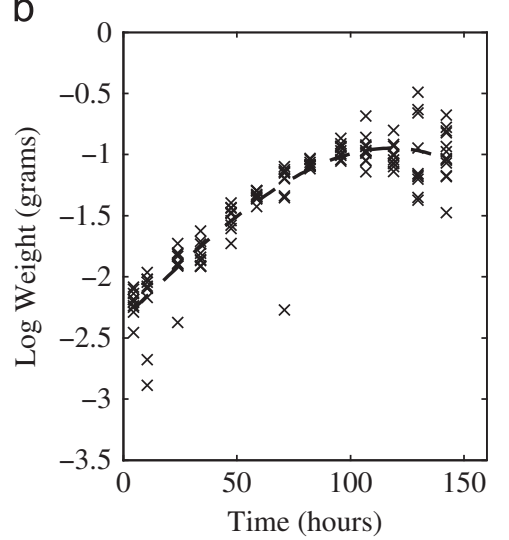

e

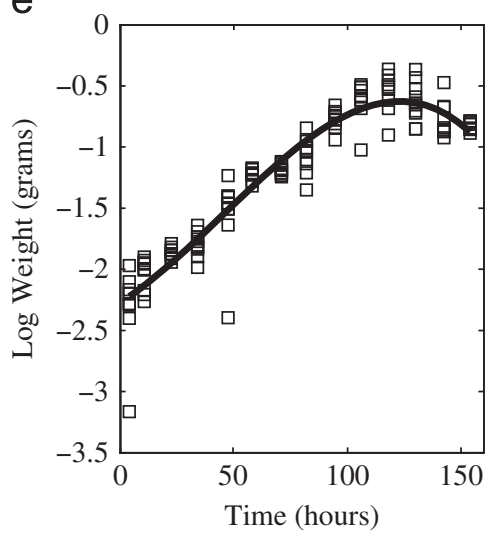

C

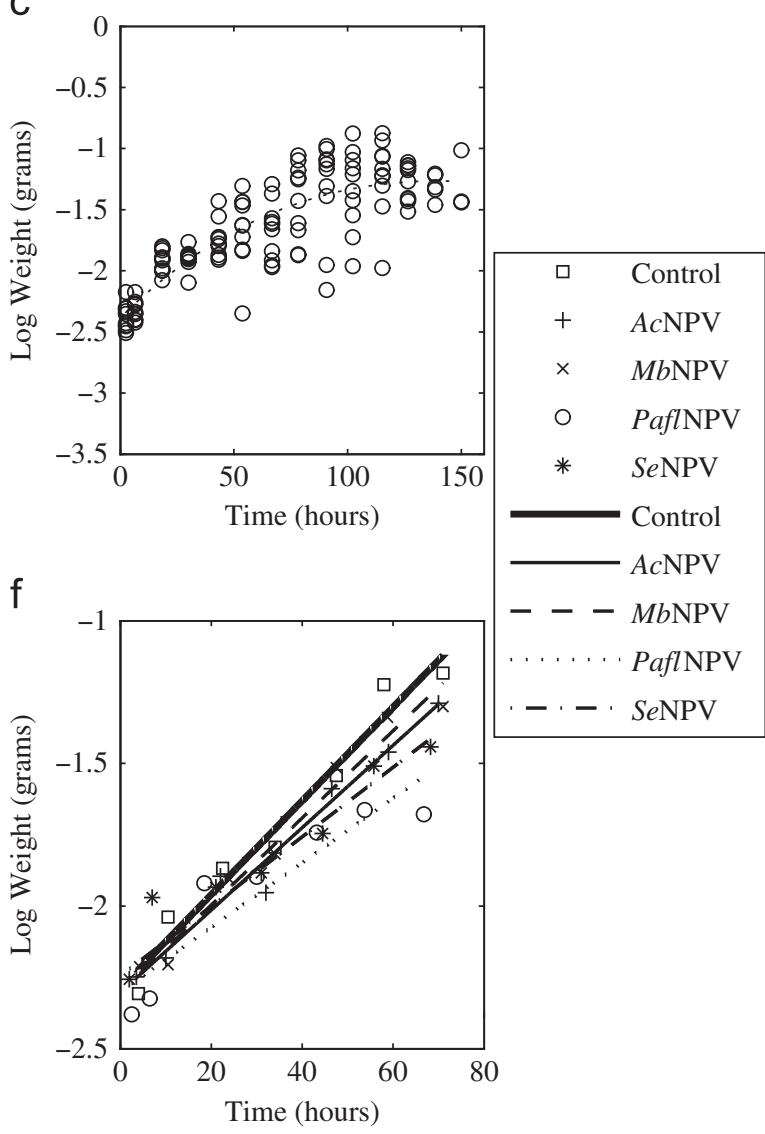

Fig. 1. Mean weight of third instar larvae infected with Ac NPV, Mb NPV, Pafl NPV, Se NPV and uninfected control. The lines show the fitted values for (a) Ac NPV $\left(\log\right.$ weight $=-2.2827+0.0104 \times$ time $+1.4605 \times 10^{-4} \times$ time $^{2}-1.3546 \times 10^{-6} \times$ time $\left.^{3}\right)$, (b) Mb NPV (log weight $=-2.3446+0.0180 \times$ time $^{-} 4.9347 \times 10^{-6} \times$ time $^{2}-$ $4.0265 \times 10^{-7} \times$ time $^{3}$ ), (c) Pafl NPV (log weight $=-2.3832+0.0183 \times$ time $-9.0041 \times 10^{-5} \times$ time $^{2}+1.1677 \times 10^{-7} \times$ time $\left.^{3}\right),(\mathrm{d})$ Se NPV $(\log$ weight $=-2.2662+$ $0.0146 \times$ time $-4.7435 \times 10^{-5} \times$ time $^{2}+1.8663 \times 10^{-7} \times$ time $^{3}$ ) and (e) uninfected controls (logweight $=-2.2718+0.0119 \times$ time $+\quad 1.3297 \times 10^{-4} \times$ time $^{2}-9.8279 \times 10^{-7} \times$ time $^{3}$ ). In (f) the mean weights are plotted for the various treatments, along with fitted lines for uninfected controls $($ log weight $=-2.2829+$ $0.01644 \times$ time $), A c$ NPV $(\log$ weight $=-2.2973+0.01444 \times$ time $), M b$ NPV $($ log weight $=-2.2963+0.01527 \times$ time $)$, Pafl NPV $($ log weight $=-2.2947+0.01131 \times$ time $)$ and Se NPV (logweight $=-2.2392+0.01219 \times$ time).

In addition, we assume that as the virus converts an increasing proportion of host mass the infection rate decreases, and tends to zero as the virus proportion approaches a maximal limit, $p$. We model this by the functional form

$\beta(t)=\beta_{0}\left(1-\frac{V(t)}{p(H(t)+V(t))}\right)$

where $\beta_{0}$ is the maximum infection rate. This assumption essentially acts to impose a 'carrying capacity' for the pathogen growth within the host since the virus growth is limited by the size of the growing host. Hence, the 'carrying capacity' is not a fixed parameter, but is dynamic with respect to the interactions between the host and the virus. Note that the parameter $p$ acts as an upper limit for the proportion of virus mass within the infected host, as we demonstrated empirically in Section 2.2.2. This is included as not all of the available host mass may be infected (the host head capsule for example), and therefore it is necessary to prescribe this limit via a reduction in the potential carrying capacity. In addition, it should also be noted that since $H(0) \gg V(0)$ it follows that $\beta(t) \geq 0$ for all $t \geq 0$.

Linear stability analysis (see Appendix A) of Model (1) reveals that there are an infinite number of locally stable equilibria which lie on the curve

$\frac{V}{p(H+V)}=1$.
Thus, for given growth parameters, $r_{0}, \beta_{0}$ and $a$, the equilibria obtained will crucially depend on the mass of the host and the virus dose at the time of infection.

\subsection{Parameter fitting}

To fit the within-host infection Model (1) to the within-host virus growth data one must convert the proportion of virus data into virus mass data. To do this we assume that the fraction of sampled DNA that is virus DNA is equal to the fraction of total host mass that is virus mass. Thus mathematically we have

mass of virus DNA at time $t \quad$ mass of virus at time $t$ $\overline{\text { total mass of DNA at time } t}=\overline{\text { total mass of infected host at time } t}$.

Using the host and virus mass data we are able to fit the within-host infection Model (1) to the data and find the associated parameter values (see Appendix B for details). Here, we use some asymptotic properties of the model to find initial estimates of the model parameters and then all parameters are found simultaneously using the initial estimates. Note that we do not prescribe $r_{0}$ from the control data. Instead we find $r_{0}$ from the simultaneous fitting on the infected data, which therefore takes into account the stochastic differences between treatments, thus allowing $r_{0}$ to emerge from the treatment data. A discussion on prescribing $r_{0}$ can be found in the ESM and Table S1. The fitted 

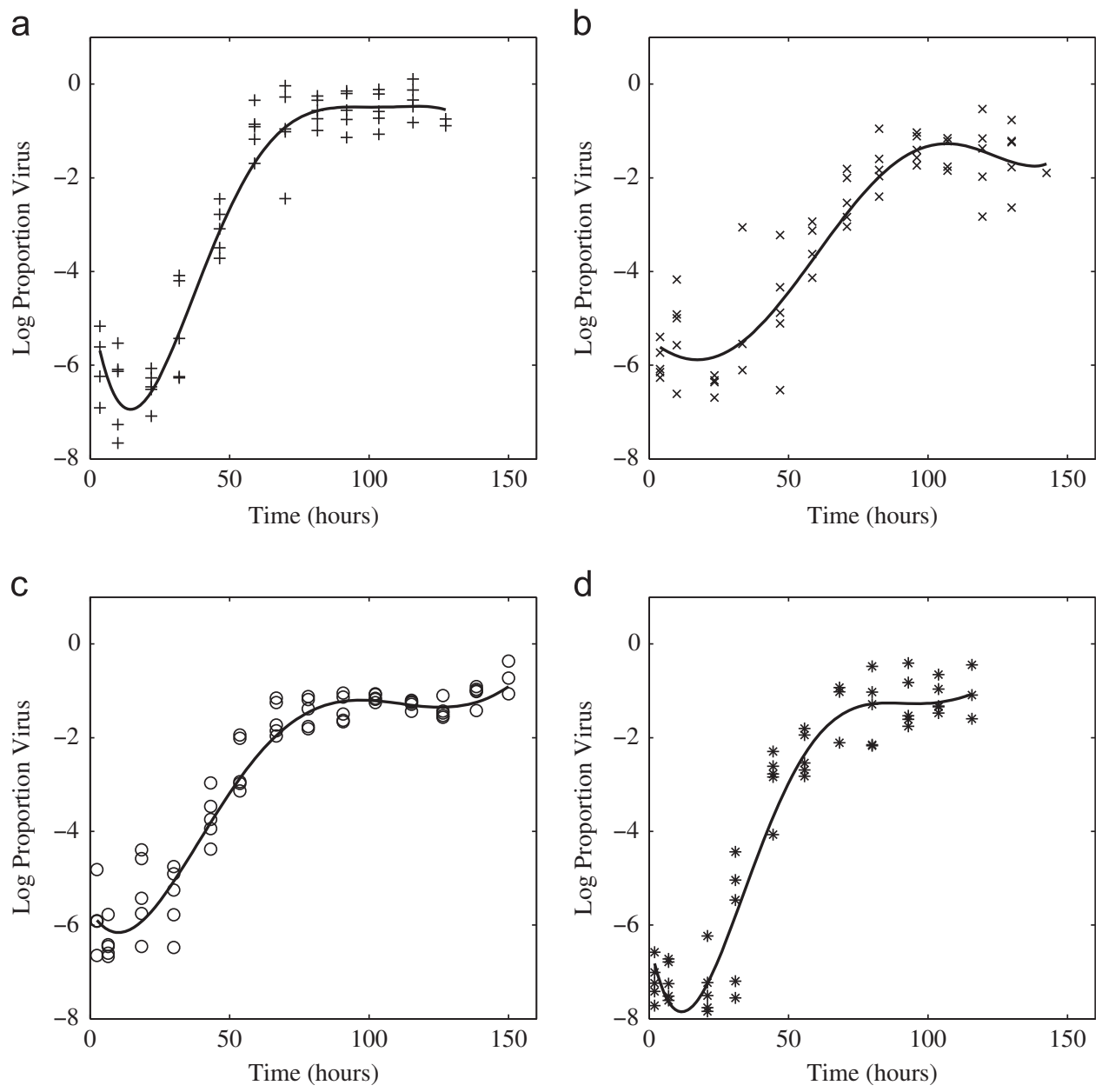

Fig. 2. The within-host growth of (a) Ac NPV, (b) Mb NPV, (c) Pafl NPV and (d) Se NPV in third instar larvae of S. exigua as measured by the proportion of total DNA represented by viral DNA. The lines show the fitted values for Ac NPV (logproportion $=-4.6000-0.3706 \times$ time $+0.0181 \times$ time $^{2}-2.7824 \times 10^{-4} \times$ time $^{3}+$ $1.8302 \times 10^{-6} \times$ time $^{4}-4.4369 \times 10^{-9} \times$ time $^{5}$ ), Mb NPV (logproportion $=-5.4454-0.0473 \times$ time $+0.0010 \times$ time $^{2}+2.0390 \times 10^{-5} \times$ time $^{3}-3.3223 \times 10^{-7} \times$ time $^{4}+$ $1.1557 \times 10^{-9} \times$ time $^{5}$ ), Pafl NPV (logproportion $=-5.6825-0.1018 \times$ time $^{2} 0.0062 \times$ time $^{2}-8.3174 \times 10^{-5} \times$ time $^{3}+4.3875 \times 10^{-7} \times$ time $^{4}-8.0036 \times 10^{-10} \times$ time $^{5}$ ) and Se NPV (logproportion $=-6.2862-0.2996 \times$ time $^{2} 0.0172 \times$ time $^{2}-2.8257 \times 10^{-4} \times$ time $^{3}+1.9346 \times 10^{-6} \times$ time $^{4}-4.8002 \times 10^{-9} \times$ time $^{5}$ ).

parameter values are listed in Table 1 and we compare the results of this parameter fitting with the data graphically in Fig. 3.

From Fig. 3 we see that Model (1) is an excellent fit to both the host and virus data, and we are able to capture all of the growth behaviour. All fits produce the characteristic log-sigmoidal virus growth and the sigmoidal growth of the host. However, due to the exceptionally fast speed of kill of Se NPV, the deceleration of host growth is almost negligible, which is reflected in the low value of $a$. In contrast, $M b$ NPV has the largest larvae at the time of death and thus the largest value for $a$. Pafl NPV and Se NPV have the largest infection rates, $\beta_{0}$, whilst $M b$ NPV has the smallest infection rate. The extent to which viruses can convert healthy host tissue into virus particles greatly differs between strains, ranging from approximately $2.8 \%$ for $M b$ NPV to as much as $22 \%$ for Ac NPV. The maximum host growth rate, $r_{0}$, does not change considerably between virus treatments. Moreover, the differences we observe in the estimation of the initial masses, $H_{0}$ and $V_{0}$, are largely due to experimental variation.

\subsection{Model predictions}

Using the fitted parameter values we can use Model (1) to predict the effects of varying the initial dose of virus and the size of the host at the time of infection.
In Fig. 4(a) we see that an increase in virus dose leads to a reduction in the yield of virus and host size at the time of host death. At first, this may seem counterintuitive, as one might expect that an increased dose may lead to an increased yield. However, the mechanism behind this phenomenon is a combination of two processes. Firstly, an increased dose has a greater initial negative effect on the host growth rate, resulting in smaller hosts, and therefore the dynamic virus 'carrying capacity' is reduced. Secondly, a greater viral dose increases the initial infection rate and therefore the virus infects a larger proportion of host more quickly, thus causing a decreased yield at death. Furthermore, extensive parameter variation, such as initial host size and host growth rate (not presented here), suggests that this is ubiquitous under our model assumptions.

In Fig. 4(b) we see the effect of varying the size of the host at the time of initial infection. As one might expect, in most cases, as host size increases, the virus yield and the size of host at the time of death both increase. This is because the size increase simply acts as an increased virus carrying capacity and there is a longer period for the virus to replicate before it has a large negative effect on the host growth rate. A similar scenario occurs when the maximum host growth rate is increased, corresponding to an increase in the host diet or environmental quality (Fig. 4(c)).

The effects of varying virus parameters can be seen in Fig. 4(d) and (f). In Fig. 4(d) we see that, as one might expect, increasing 
Table 1

Fitted parameter values for the infection model using the method outlined in Section 3.2 for the total host mass and within-host mass growth of Ac NPV, Mb NPV, Pafl NPV and Se NPV in third instar larvae of S. exigua. The bracketed numbers correspond to the 95\% bootstrapped confidence interval for each parameter.

\begin{tabular}{|c|c|c|c|c|}
\hline Parameter & Ac NPV & Pafl NPV & $M b$ NPV & Se NPV \\
\hline Initial host mass $(\mathrm{g}), H_{0}$ & $\begin{array}{l}5.0642 \times 10^{-3} \\
\left(4.4941 \times 10^{-3}, 5.6508 \times 10^{-3}\right)\end{array}$ & $\begin{array}{l}5.6441 \times 10^{-3} \\
\left(4.0462 \times 10^{-3}\right. \\
\left.6.3307 \times 10^{-3}\right)\end{array}$ & $\begin{array}{l}5.9695 \times 10^{-3} \\
\left(4.5804 \times 10^{-3}\right. \\
\left.7.7104 \times 10^{-3}\right)\end{array}$ & $\begin{array}{l}4.3949 \times 10^{-3} \\
\left(3.7634 \times 10^{-3}, 4.5878 \times 10^{-3}\right)\end{array}$ \\
\hline Virus dose $(\mathrm{g}), V_{0}$ & $\begin{array}{l}1.3089 \times 10^{-9} \\
\left(7.2990 \times 10^{-10}\right. \\
\left.3.3345 \times 10^{-9}\right)\end{array}$ & $\begin{array}{l}3.5492 \times 10^{-9} \\
\left(1.9431 \times 10^{-9}\right. \\
\left.6.9817 \times 10^{-8}\right)\end{array}$ & $\begin{array}{l}9.1399 \times 10^{-9} \\
\left(5.6659 \times 10^{-9},\right. \\
\left.2.1487 \times 10^{-8}\right)\end{array}$ & $\begin{array}{l}2.4957 \times 10^{-10} \\
\left(1.7004 \times 10^{-10}\right. \\
\left.5.1954 \times 10^{-10}\right)\end{array}$ \\
\hline Max. host growth rate $\left(\mathrm{h}^{-1}\right), r_{0}$ & $\begin{array}{l}3.7446 \times 10^{-2} \\
\left(3.4601 \times 10^{-2}, 4.2140 \times 10^{-2}\right)\end{array}$ & $\begin{array}{l}2.3584 \times 10^{-2} \\
\left(2.0334 \times 10^{-2}\right. \\
\left.3.2734 \times 10^{-2}\right)\end{array}$ & $\begin{array}{l}3.4451 \times 10^{-2} \\
\left(2.8663 \times 10^{-2}\right. \\
\left.4.1988 \times 10^{-2}\right)\end{array}$ & $\begin{array}{l}3.6758 \times 10^{-2} \\
\left(3.4622 \times 10^{-2}, 3.7876 \times 10^{-2}\right)\end{array}$ \\
\hline Zero infection virus proportion, $p$ & $\begin{array}{l}2.1588 \times 10^{-1} \\
\left(1.3742 \times 10^{-1}, 3.5856 \times 10^{-1}\right)\end{array}$ & $\begin{array}{l}4.9161 \times 10^{-2} \\
\left(3.8357 \times 10^{-2}\right. \\
\left.6.5778 \times 10^{-2}\right)\end{array}$ & $\begin{array}{l}2.8096 \times 10^{-2} \\
\left(1.6406 \times 10^{-2}\right. \\
\left.4.3981 \times 10^{-2}\right)\end{array}$ & $\begin{array}{l}4.6904 \times 10^{-2} \\
\left(4.5363 \times 10^{-2}, 1.7088 \times 10^{-1}\right)\end{array}$ \\
\hline Max. infection rate $\left(\mathrm{g}^{-1} \mathrm{~h}^{-1}\right), \beta_{0}$ & $\begin{array}{l}16.750 \\
(13.760,17.022)\end{array}$ & $\begin{array}{l}16.530 \\
(14.142,21.722)\end{array}$ & $\begin{array}{l}6.9231 \\
(5.4750,7.7177)\end{array}$ & $\begin{array}{l}20.151 \\
(16.354,21.331)\end{array}$ \\
\hline $\begin{array}{l}\text { Host growth reduction rate }\left(\mathrm{h}^{-1}\right) \text {, } \\
a\end{array}$ & $\begin{array}{l}1.9390 \\
(0.9933,4.5449)\end{array}$ & $\begin{array}{l}7.9301 \\
(2.3150,22.454)\end{array}$ & $\begin{array}{l}31.813 \\
(20.025,128.84)\end{array}$ & $\begin{array}{l}5.0346 \\
(1.2807,5.5024)\end{array}$ \\
\hline
\end{tabular}
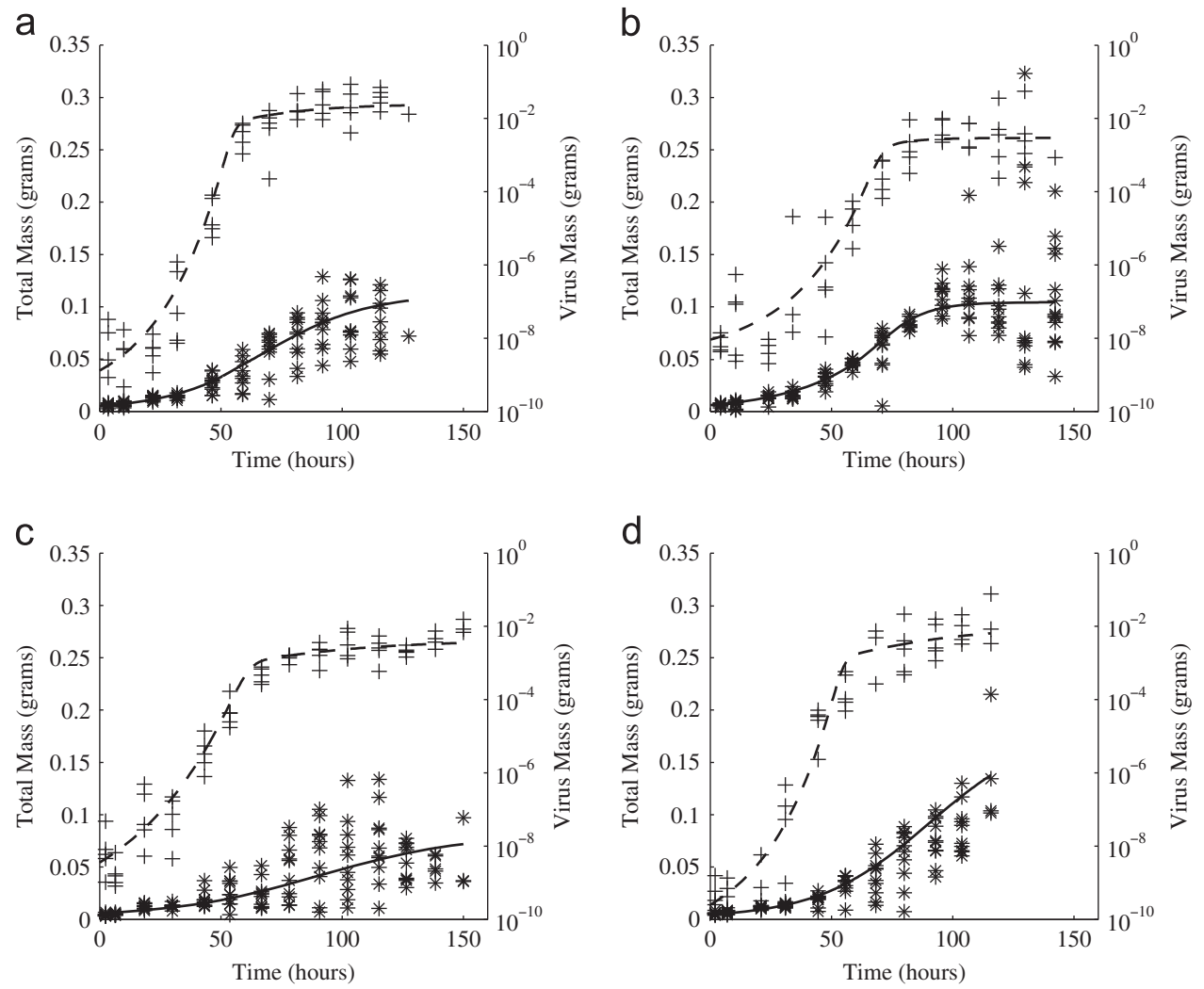

Fig. 3. The total host mass and within-host mass growth of (a) Ac NPV, (b) Mb NPV, (c) Pafl NPV and (d) Se NPV in third instar larvae of S. exigua. The asterisks denote total host mass, plus signs denote virus mass from the experimental data and the solid and dashed lines show the results of the fitted Model (1) for the parameter values in Table 1 . Note that the virus axes are in a $\log _{10}$ scale.

the rate at which the host growth is reduced by the virus infection causes a decrease in host mass which in turn reduces the virus yield. In contrast, one might expect that increasing the infection rate of the virus would increase the virus yield, however, in Fig. 4(e) we see that the opposite is true. This is due to the increase in maximum infection rate $\left(\beta_{0}\right)$ causing the virus mass to utilise more of the host mass more quickly, resulting in earlier saturation. Therefore, the host is increasingly moribund and suffers from a reduction in growth rate, final host mass and hence a reduction in virus yield. Finally, in Fig. 4(f) we show the effect of varying the zero infection virus proportion, $p$. Intuitively, we see an increase in proportion of host that the virus can infect causes an increase in viral yield, which in turn reduces the host mass since the host growth rate is reduced by the additional virus mass.

\section{Conclusions \& discussion}

It is well known that genetically similar virus strains show differences in pathogenicity, speed of kill and yield (Hodgson et al., 2001), but here we have shown that they also differ in how they may impact host growth and replicate within it. We have demonstrated empirically that virus infection impedes the growth rate of the host, with some viruses doing so from the 

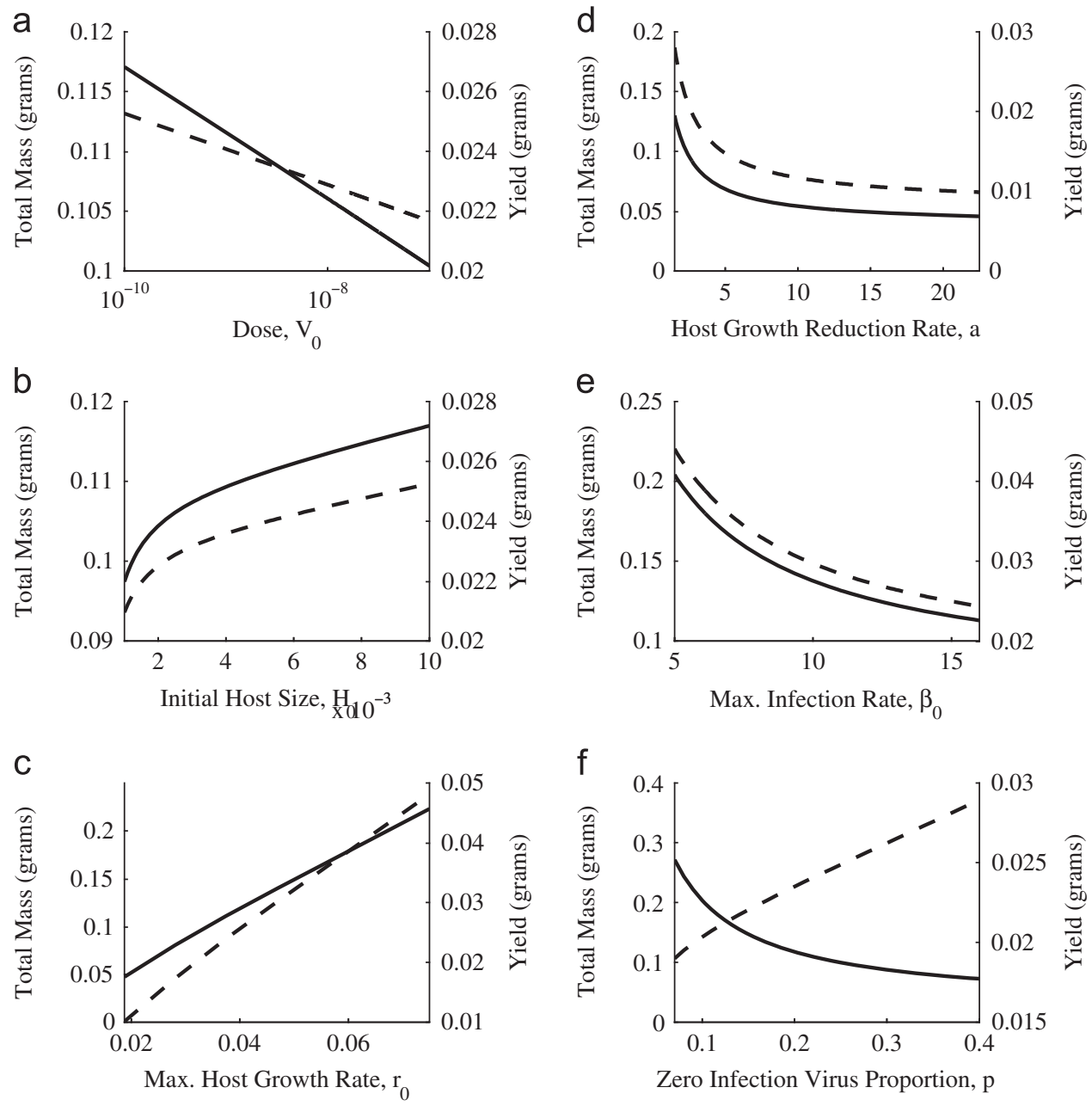

Fig. 4. The effects of the virus parameters on infections. Here we run simulations of Model (1) using the parameters in Table 1 for Ac NPV. In each graph the total host mass (solid line) and the virus yield (dashed line) at the time of host death is plotted against (a) virus dose, $V_{0}$, (b) initial host mass, $H_{0}$, (c) maximum host growth rate, $r_{0}$, (d) host growth reduction rate, $a$, (e) maximum infection rate, $\beta_{0}$, and (f) zero infection virus proportion, $p$. The time to death is 160 h. Note that qualitatively similar results hold for $\mathrm{Mb}$ NPV, Se NPV and Pafl NPV.

early stages of infection, and that this inhibition increases as the infection progresses and has consequences for the outcome of infection. Four genotypically similar strains of pathogen were found to have differences in traits associated with pathogen fitness (speed of kill, pathogencity), and to impede host growth to differing degrees (parameter $a$ varied by more than an order of magnitude). This led to a lack of correlation between the standard phenotypic traits (speed of kill, pathogenicity) and the efficiency with which the different viruses converted host tissue to virus (for which Ac NPV had the highest ratio of virus DNA to host DNA and $M b$ NPV had the lowest).

Using the fitted parameter values from the within-host infection model, we have seen that Ac NPV is relatively slow at infecting healthy host tissue. On the other hand, it is clear from the fitted parameter values and the simulations that Se NPV is the fastest growing virus, but it does not convert a high ratio of host mass into virus mass. From these parameter-fitting results we can conclude that there is a lack of correlation between initial virus growth rate $\left(\beta_{0}\right)$ and both the speed of kill and mortality. For example, Se NPV does have the highest initial growth rate and it also has the fastest speed of kill and mortality. In contrast, Pafl NPV has the slowest speed of kill and lowest mortality, but does not have the lowest initial virus growth rate (this belongs to $M b$ NPV). This would suggest that one cannot predict speed of kill, mortality and virus yield from initial virus growth rate alone and that these pathogen fitness parameters are a result of a number of interacting processes.

Surprisingly, empirical investigation into parasite growth rates has been largely neglected in invertebrate hosts (Hamilton et al., 2008). Our experimental data highlight the initial fast speed at which virus replication occurs. Using an approximation of our mathematical model (B.3), we have shown that the initial virus growth rate is double exponential $\left(V(t) \approx e^{e^{t}}\right)$. This is in stark contrast to previous theory of obligate killing parasites, where more simplistic logistic growth curves have been assumed (Ebert and Weisser, 1997), thus underestimating initial virus growth. Indeed, for the prodigious theory of human diseases the withinhost growth of viruses is often shown to be significantly slower $\left(V(t) \approx e^{t}\right)$ (Ribeiro et al., 2010), even before innate or adaptive immune responses slow the within-host spread of disease. This further highlights the differences between the complexity of vertebrate and simplicity of invertebrate hosts and their diseases. The main reason for this difference is the speed at which the host grows. In vertebrate systems host growth is assumed to be constant, since the speed of replication of the pathogen is much faster than the growth rate of the host (Steinmeyer et al., 2010 for example).

Interestingly, the fitted statistical model (Fig. 2) shows a decline in virus abundance for each of the virus genotypes at approximately $10-20 \mathrm{~h}$ post infection. Why this occurs remains 
unclear. A possible explanation is a sloughing defence mechanism (Keddie et al., 1989) or simply loss of virus particles on the outer body of the larva from droplet feeding, which illustrates the sensitivity of the molecular method used. It may also be attributed to more complex cellular and humoral mechanisms of immunity and both have been implicated in insect resistance to baculoviruses (Washburn et al., 1996). In terms of our results, the decline of the virus abundance is likely to have some small effect on the fitted parameter values, in particular underestimating the $\beta_{0}$ values. Further study is clearly required to ascertain the precise cause of the reduction in virus abundance at the early stages of an overt infection and to understand the implications for the host and virus growth dynamics.

From our observations we hypothesise that the speed of kill is strongly dependent on the rate at which the virus grows within the host, how quickly the virus replication rate reaches a plateau (if at all) and how much the virus impedes host growth, but the exact relationship is not immediately obvious. For example, Pafl NPV has the slowest speed of kill, whilst in contrast Mb NPV has the smallest maximum infection rate, $\beta_{0}$. Therefore, using a simple single parameter to predict the speed of kill is not possible. Furthermore, the biological mechanism behind host death (i.e. timing of host death relative to infection levels) is still relatively undetermined. Previous models have assumed that host death occurs when pathogen fitness is maximised (Ebert and Weisser, 1997). Ebert and Weisser (1997) assumed that the fitness of the obligately killing pathogen, $F$, is given by

$F\left(t_{\text {kill }}\right)=V\left(t_{\text {kill }}\right) e^{-m t_{\text {kill }}}$

where $V\left(t_{\text {kill }}\right)$ is the number of transmission stages at the time of host death, $t_{\text {kill, }}$ and $m$ is the background host mortality. Maximising (3) with respect to the time of host death gives the optimal speed of kill. Ebert and Weisser (1997) found that under their model assumptions for the within-host virus growth the optimal killing time approximately corresponded to the period of time during which viral replication rate significantly decreases. However, applying this optimisation to our within-host viral growth model, parameterised for our four strains of NPV, results in nonsensical optimal speeds of kill, even for a wide range of background mortalities. This suggests that the speed of kill of baculovirus infections may be more complex than simple pathogen fitness optimisation or that pathogen fitness is not suitably described by (3). Moreover, our empirical data do not support the finding that the killing time occurs at a point of rapid deceleration in the viral replication rate. In contrast, we find that rapid viral growth, which has been shown in many host-pathogen systems (Otterstatter and Thomson, 2006), is followed by a prolonged period of deceleration towards a stationary final viral mass (see also Evans et al., 1981). However, this deceleration is less pronounced for some viral strains, in particular Se NPV.

To this end, in the ESM we have covaried the speed of kill alongside the other model parameters for two contrasting strains: Ac NPV and Se NPV, where the former exhibits a strong saturation effect. In each case, a faster speed of kill leads to reduced viral yield and small hosts at the time of death, as one would expect. However, for Ac NPV, some parameters are more sensitive to the speed of kill than others - the most sensitive parameters being the initial host mass and viral dose parameters. In contrast, for Se $\mathrm{NPV}$, the speed of kill has a large effect on host mass at the time of death and the viral yield for each parameter variation. Therefore, we must conclude that for viruses that exhibit weak saturation, the speed of kill will have a large effect on the viral yield. Moreover, if the speed of kill is greatly altered by either (i) inoculating different insect instars or (ii) changing the viral dose concentration, then viral yield will be greatly affected. Conversely, if the host's environment can affect other model parameters, resulting in different speeds of kill, then there may be no significant change in the viral yield.

Our results show that pathogen infection slows the growth rate of the host, even at the early stages of infection. Surprisingly, there are relatively few studies that empirically demonstrate a reduction in host growth rate (but see Burand and Park, 1992), but this is often suggested since parasites cause harm to their hosts as an unavoidable consequence of parasite reproduction. Our model predicts that a greater virus dose will increase this effect, which agrees with evidence from a similar host-pathogen system (Burand and Park, 1992). To date, the exact reason behind the reduction of host growth rate is unknown, but a number of possible mechanisms have been put forward. These include hostvirus competition for nutrients at the cellular level (Burand and Park, 1992) and the expression of the viral ecdysteroid UDPglucosyl transferase (EGT) gene which alters host hormones related to host development (Wilson et al., 2000). Our model does not explicitly state the origins of the growth rate reduction, but we simply incorporate this effect as a composition parameter on the host-virus growth dynamics, which produces a good fit. This enables us to detect differences between virus strains and thus yields, which in terms of transmission in the field is critical, as yield has a direct effect on the abundance of overwintering inoculum.

Our model predicts that larger hosts at the time of infection result in larger viral yields, which supports experimental results of others (Evans et al., 1981). Counterintuitively, our model reveals that larger viral doses may decrease viral yield. This result has been discovered experimentally in other closely related systems (Evans et al., 1981; Hodgson et al., 2002), but this is not always the case (Cory et al., 2005), perhaps due to the tradeoffs between dose, speed of kill and virus yield obscuring this phenomenon. In terms of maximising transmission, the virus will increase its yield with lower doses, but this will trade-off against the probability of infection. Therefore, transmission is likely to be maximised for some intermediate dose. Our result also contrasts with vertebrate within-host theory, where Steinmeyer et al. (2010) found that increasing viral dose increased the peak viral load, whilst empirical evidence suggests the contrary, as found in sheep inoculated intranasally with a type $O$ foot-and-mouth disease virus (Hughes et al., 2002). Here the authors suggested the reason for this is that cell-mediated immune mechanisms responded more quickly to high doses than lower doses, the result being increased inhibition of viral replication.

It has been suggested that environmental stress increases host susceptibility to infections and reduces host ability to resist parasite growth and reproduction, thus benefiting parasites. This suggestion stems from expected costs of immune defence; hosts in poor condition should have fewer resources to be allocated to immune function. However, the alternative hypothesis for the response to environmental stress is that hosts in poor condition provide fewer resources for parasites and/or suffer higher mortality, leading to reduced parasite growth, reproduction and survival (Seppälä et al., 2008). Under the assumption that poorer quality diet results in a lower host growth rate $\left(r_{0}\right)$, our model predicts a reduction in virus yield, and so supports the latter hypothesis.

Despite the focus of most host-pathogen work concentrating on single infections, as we have studied here, molecular techniques have revealed that many infections in insect hosts are caused by several pathogen genotypes which differ phenotypically in their interaction with the host (Hodgson et al., 2001). One example is the pine beauty moth, Panolis flammea, in which a plethora of genetically distinct strains of NPVs have been isolated from a single host. These strains have been found to differ phenotypically in parameters correlated with fitness, including 
the speed with which the pathogen kills the host and the subsequent yields of OBs (Hodgson et al., 2001), which may act as non-lethal synergists by interacting with secondary virus strains but are not themselves lethal (Bonsall, 2010). The simplest assumption would be that competition between genotypes within a host is a race to gain the greatest share of resources (host tissues) (May and Nowak, 1995), as in the tragedy of the commons (Hardin, 1968). As a consequence, mixed infections may lead to reduced transmission between hosts. Hence, understanding within-host dynamics of multiple infections is essential for understanding the impact of multiple pathogens in the field.

\section{Acknowledgments}

This work was supported by an MRC Discipline Hopping Award G0300571 to PKM and RSH. PKM was partially supported by a Royal Society Wolfson Research Merit Award. SMW would like to thank Stephen J. Cornell and Greg Dwyer for some useful discussions, and Tim Carty for providing the insects and diet.

\section{Appendix A. Model analysis}

Since $r(t) \rightarrow 0$ as $t \rightarrow \infty$ the nullclines are given by $\beta(t)=0$ for both $H^{\prime}=V^{\prime}=0$, and thus are given by

$\frac{V}{p(H+V)}=1$.

Hence, the nullclines for both of the coupled differential equations completely overlap and therefore the equilibria, which are given by the intersection of the nullclines, are defined by a curve.

Proof of our claims on stability will be reported elsewhere; here we simply sketch the details. Straightforward linear stability analysis reveals the existence of a centre manifold. The long-term behaviour critically depends on the initial conditions. The system will blow up if the initial dose is sufficiently large relative to the initial host size, that is, if $\beta(0)<0$. However, given that the virus dose is small compared to the initial host size it is biologically reasonable to assume that $\beta(0)>0$. Then, since the equilibrium is given by $\beta(t)=0$ it can be shown that the system tends to the equilibrium. Moreover, the fast and slow dynamics are calculable for the manifold.

\section{Appendix B. Parameter fitting}

Here we outline the method used for the parameter fitting of the mathematical model (1). The results of these methods are listed in Table 1 and shown graphically in Fig. 3. For this we use a two-stage process:

1. Since we have assumed that in the absence of virus the host initially grows exponentially, we fit the curve

$H(t)=H(0) e^{r_{0} t}$

where $H(0)=H_{0_{\text {cont }}}$, to the initial growth phase of the control data, thus giving the initial estimate of the maximum growth rate $r_{0}$ and the initial host mass $H_{0_{\text {cont }}}$ for the control data. We then use $r_{0}$ and the total mass of the infected hosts data to find the initial size of the host, $H_{0}$.

To find the initial estimates of the maximum infection rate, $\beta_{0}$, and the initial dose, $V_{0}$, we use the fact that initially the amount of virus within the host is small. Hence we may approximate model (1) by

$\mathrm{d} H$

$\frac{\mathrm{d} H}{\mathrm{~d} t}=r_{0} H$

$\frac{\mathrm{d} V}{\mathrm{~d} t}=\beta_{0} H V$

Solving (B.2) we obtain

$V(t)=V_{0} \exp \left\{\frac{\beta_{0} H_{0}}{r_{0}}\left(e^{r_{0} t}-1\right)\right\}$

where $V(0)=V_{0}$, for the initial growth of the virus. Hence one can use (B.3) to fit to the initial part of the virus data to find the initial parameter estimates for $\beta_{0}$ and $V_{0}$.

It is not possible to find initial estimates for the host growth reduction rate, $a$, and the zero infection virus proportion, $p$, using techniques similar to those above. Therefore, we fit the model to both the total host mass and virus mass data simultaneously by making use of the previously found initial parameter estimates.

2. The set of six initial parameter estimates are then fitted to the data simultaneously where the previously found parameter estimates are used as 'good' initial guesses. Since the data exhibit growing variance over the course of the infection, we perform a log-transformation to account for this (Grafen and Hails, 2002).

All fitting is achieved by the method of least squares using a modified Levenberg-Marquardt algorithm (Fletcher, 1971) and implemented in MATLAB ${ }^{\circledR}$, using a Runge-Kutta method for solving the differential equations numerically. This fitting method, often referred to as "trajectory matching" (Bolker, 2008) or "model calibration" (Kendall et al., 1999), has been successful in fitting in other biological datasets (see Harrison, 1995 for example). Our method works well here since the timeseries has little process noise and we assume that all the error is from observation, but for noisy data more complex methods can be used, such as a gradient matching method (Ellner et al., 2002).

The bootstrapped confidence intervals are calculated from 10,000 bootstrapped datasets (with replacements), to which the model is fitted using the parameter estimates as initial guesses.

\section{Appendix C. Supplementary data}

Supplementary data associated with this article can be found in the online version at http://dx.doi.org.10.1016/j.jtbi.2012.07.022.

\section{References}

Alizon, S., van Baalen, M., 2008. Multiple infections, immune dynamics, and the evolution of virulence. Am. Nat. 172, E150-E168.

Antia, R., Lipsitch, M., 1997. Mathematical models of parasite responses to host immune defences. Parasitology 115, S155-S167.

Antia, R., Levin, B.R., May, R.M., 1994. Within-host population dynamics and the evolution and maintenance of microparasite virulence. Am. Nat. 144, 457-472.

Ayres, M.D., Howard, S.C., Kuzio, J., Lopez-Ferber, M., Possee, R.D., 1994. The complete DNA sequence of Autographa californica nuclear polyhedrosis virus. Virology 202, 586-605.

Blanford, S., Chan, B.H.K., Jenkins, N., Sim, D., Turner, R.J., Read, A.F., Thomas, M.B., 2005. Fungal pathogen reduces potential for malaria transmission. Science 308, 1638-1641.

Bolker, B.M., 2008. Ecological Models and Data in R. Princeton University Press.

Bonsall, B.M., 2010. The impact of non-lethal synergists on the population and evolutionary dynamics of host-pathogen interactions. J. Theor. Biol. 262 567-575.

Borer, E.T., Hosseini, P.R., Seabloom, E.W., Dobson, A.P., 2007. Pathogen-induced reversal of native dominance in a grassland community. Proc. Natl. Acad. Sci. USA $104,5473-5478$. 
Burand, J.P., Park, E.J., 1992. Effect of nuclear polyhedrosis virus infection on the development and pupation of gypsy moth larvae. J. Invertebr. Pathol. 60, 171-175.

Burden, J.P., Possee, R.D., Sait, S.M., King, L.A., Hails, R.S., 2006. Phenotypic and genotypic characterisation of persistent baculovirus infections in populations of the cabbage moth (Mamestra brassicae) within the british isles. Arch. Virol. $151,635-649$.

Cory, J.S., Green, B.M., Paul, R.K., Hunter-Fujita, F.R., 2005. Genotypic and phenotypic diversity of a baculovirus population within an individual insect host. J. Invertebr. Pathol. 89, 101-111.

Daszak, P., Cunningham, A.A., Hyatt, A.D., 2000. Emerging infectious diseases of wildlife - threats to biodiversity and human health. Science 287, 443-449.

de Roode, J.C., Pedersen, A.B., Hunter, M.D., Altizer, S., 2008. Host plant species affects virulence in monarch butterfly parasites. J. Anim. Ecol. 77, 120-126.

Dwyer, G., Dushoff, J., Elkinton, J.S., Levin, S.A., 2000. Pathogen-driven outbreaks in forest defoliators revisited: building models from experimental data. Am. Nat. 156, 105-120.

Ebert, D., Weisser, W.W., 1997. Optimal killing for obligate killers: the evolution of life histories and virulence of semelparous parasites. Proc. R. Soc. B 264, 985-991.

Ellner, S.P., Seifu, Y., Smith, R.H., 2002. Fitting population dynamic models to timeseries data by gradient matching. Ecology 83, 2256-2270.

Ellner, S.P., Jones, L.E., Mydlarz, L.D., Harvell, C.D., 2007. Within-host disease ecology in the sea fan Gorgonia ventalina: modeling the spatial immunodynamics of a coral-pathogen interaction. Am. Nat. 170, E143-E161.

Evans, H.F., Lomer, C.J., Kelly, D.C., 1981. Growth of nuclear polyhedrosis virus in larvae of the cabbage moth, Mamestra brassicae L. Arch. Virol. 70, 207-214.

Fletcher, R., 1971. A Modified Marquardt Subroutine for Non-Linear Least Squares. Technical Report AERE-R-6799, Atomic Energy Research Establishment, Harwell (England).

Grafen, A., Hails, R., 2002. Modern Statistics for the Life Sciences. Oxford University Press.

Hamilton, R., Siva-Jothy, M., Boots, M., 2008. Two arms are better than one: parasite variation leads to combined inducible and constitutive innate immune responses. Proc. R. Soc. B 275, 937-945.

Hardin, G., 1968. The tragedy of the commons. Science 162, 1243-1248.

Harrison, G.W., 1995. Comparing predator-prey models to luckinbill's experiment with didinium and paramecium. Ecology 76, 357-374.

Hodgson, D.J., Vanbergen, A.J., Watt, A.D., Hails, R.S., Cory, J.S., 2001. Phenotypic variation between naturally co-existing genotypes of a lepidopteran baculovirus. Evol. Ecol. Res. 3, 687-701.

Hodgson, D.J., Vanbergen, A.J., Hartley, S.E., Hails, R.S., Cory, J.S., 2002. Differential selection of baculovirus genotypes mediated by different species of host food plant. Ecol. Lett. 5, 512-518.

Hudson, P.J., Dobson, A.P., Newborn, D., 1998. Prevention of population cycles by parasite removal. Science 208, 2256-2258.
Hughes, P.R., Beek, N.A.M.V., Wood, H.A., 1986. A modified droplet feeding method for rapid assay of Bacillus thuringiensis and baculoviruses in noctuid larvae. J. Invertebr. Pathol. 48, 187-192.

Hughes, G.J., Kitching, R.P., Woolhouse, M.E.J., 2002. Dose-dependent responses of sheep inoculated intranasally with a type o foot-and-mouth disease virus. J. Comp. Pathol. 127, 22-29.

Hunter-Fujita, F.R., Entwistle, P.F., Evans, H.F., Crook, N.E., 1998. Insect Viruses and Pest Management. John \& Wiley Sons.

Ijkel, W.F.J., van Strien, E.A., Heldens, J.G.M., Broer, R., Zuidema, D., Goldbach, R.W., Vlak, J.M., 1999. Sequence and organisation of the Spodoptera exigua multicapsid nucleoployhedrovirus genome. J. Gen. Virol. 80, 3289-3304.

Keddie, B.A., Aponte, G.W., Volkman, L.E., 1989. The pathway of infection of Autographa californica nuclear polyhedrosis virus in an insect host. Science 243, 1728-1730.

Kendall, B.E., Briggs, C.J., Murdoch, W.W., Turchin, P., Ellner, S.P., McCauley, E., Nisbet, R.M., Wood, S.N., 1999. Why do populations cycle? A synthesis of statistical and mechanistic modeling approaches. Ecology 80, 1789-1805.

Lacey, L.A., Kaya, H.K. (Eds.), 2007. Field Manual of Techniques in Invertebrate Pathology: Application and Evaluation of Pathogens for Control of Insects and other Invertebrate Pests, second edition Springer, Netherlands.

May, R.M., Nowak, M.A., 1995. Coinfection and the evolution of parasite virulence. Proc. R. Soc. B 261, 209-215.

Mitchell, C.E., Power, A.G., 2003. Release of invasive plants from fungal and viral pathogens. Nature 421, 625-627.

Otterstatter, M.C., Thomson, J.D., 2006. Within-host dynamics of an intestinal pathogen of bumble bees. Parasitology 133, 749-761.

Perelson, A.S., 2002. Modelling viral and immune system dynamics. Nat. Rev. Immunol. 2, 28-36.

Ribeiro, R.M., Qin, L., Chavez, L.L., Li, D., Self, S.G., Perelson, A.S., 2010. Estimation of the initial viral growth rate and basic reproductive number during acute HIV-1 infection. J. Virol. 84, 6096-6102.

Schmid-Hempel, P., 2005. Evolutionary ecology of insect immune defenses. Annu. Rev. Entomol. 50, 529-551.

Seppälä, O., Liljeroos, K., Karvonen, A., Jokela, J., 2008. Host condition as a constraint for parasite reproduction. Oikos 117, 749-753.

Siva-Jothy, M.T., Moret, Y., Rolff, J., 2005. Insect immunity: an evolutionary ecology perspective. Adv. Insect Physiol. 32, 1-48.

Steinmeyer, S.H., Wilke, C.O., Pepin, K.M., 2010. Methods of modelling viral disease dynamics across the within- and between-host scales: the impact of virus dose on host population immunity. Proc. R. Soc. B 365, 1931-1941.

Washburn, J.O., Kirkpatrick, B.A., Volkman, L.E., 1996. Insect protection against viruses. Nature 383, 767.

Wilson, K.R., O’Reilly, D.R., Hails, R.S., Cory, J.S., 2000. Age-related effects of the autographa californica multiple nucleopolyhedrovirus egt gene in the cabbage looper (Trichoplusia ni). Biol. Control 19, 57-63. 